\title{
Kondo lattice mediated interactions in flat-band systems
}

\author{
Pramod Kumar $\odot$, Guangze Chen $(\mathbb{0}$, and J. L. Lado $(1)$ \\ Department of Applied Physics, Aalto University, 00076 Aalto, Espoo, Finland
}

(Received 15 July 2021; revised 27 September 2021; accepted 27 October 2021; published 12 November 2021)

\begin{abstract}
Electronic flat bands represent a paradigmatic platform to realize strongly correlated matter due to their associated divergent density of states. In common instances, including electron-electron interactions leads to magnetic instabilities for repulsive interactions and superconductivity for attractive interactions. Nevertheless, interactions of a Kondo nature in flat-band systems have remained relatively unexplored. Here we address the emergence of interacting states mediated by Kondo lattice coupled to a flat-band system. Combining dynamical mean-field theory and tensor networks methods to solve flat-band Kondo lattice models in one and two dimensions, we show the emergence of a robust underscreened regime leading to a magnetically ordered state in the flat band. Our results put forward flat-band Kondo lattice models as a platform to explore the genuine interplay between flat-band physics and many-body Kondo screening.
\end{abstract}

DOI: 10.1103/PhysRevResearch.3.043113

\section{INTRODUCTION}

Flat-band systems represent one of the paradigmatic systems to engineer correlated matter [1-6]. Quantum engineering has provided a variety of platforms potentially combining both flat bands and interactions, including atomic lattices [7-10], cold atoms [11-13], and twisted moire materials [14-16]. Their potential for correlated physics stems from the vanishing electronic dispersion, which creates a greatly enhanced density of states at the Fermi energy [3,1719]. While a wide variety of correlated states in flat-band systems can emerge, minimal attractive or repulsive onsite interactions are well known to lead to magnetism and superconductivity, respectively [2,15,20-26]. More complex interactions in flat bands are also well known to give rise to other symmetry broken states, including charge-density waves and bond orders [27-30]. The different interactions considered are usually written as an effective density-density interaction. Nevertheless, external couplings such as Kondo couplings $[31,32]$ in the system can lead to even more sophisticated interaction terms.

Electronic states coupled to magnetic impurities are known as prototypical many-body states [33,34]. The simplest example corresponds to the Kondo problem, in which a single magnetic impurity forms a many-body ground state with its electronic bath [33-35]. The lattice version of the problem, known as the Kondo lattice problem, represents the starting point for exotic physical phenomena found in heavy-fermion systems [33,36-43]. Interestingly, the Kondo physics outlined above is usually addressed in systems with strong electronic dispersion, while the Kondo problem for flat bands has been

Published by the American Physical Society under the terms of the Creative Commons Attribution 4.0 International license. Further distribution of this work must maintain attribution to the author(s) and the published article's title, journal citation, and DOI. much less explored [44-48]. In the dispersive limit, the interaction between a local magnetic impurity and the conduction bath is determined by the Kondo temperature, increasing with the density of states, and therefore divergent in the flat-band regime. The coupling between magnetic impurities, known as the Ruderman-Kittel-Kasuya-Yosida (RKKY) interaction [49-51], is determined by the Fermi wavelength of

(a)

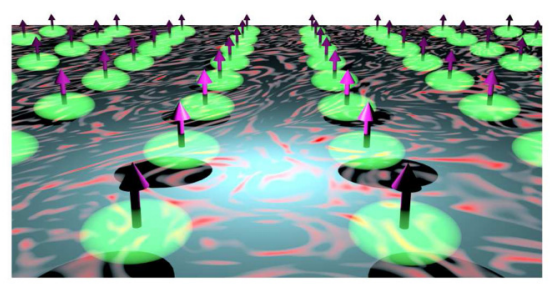

(b)

(d)
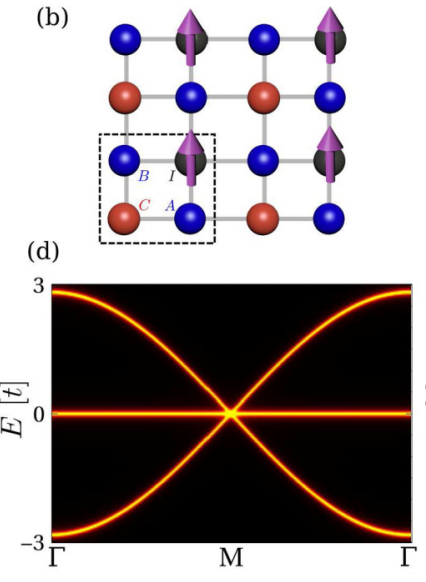

$A(\mathrm{k}, \omega)$ (c)

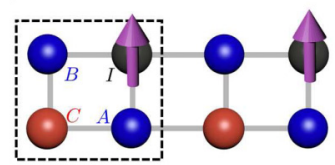

(e)

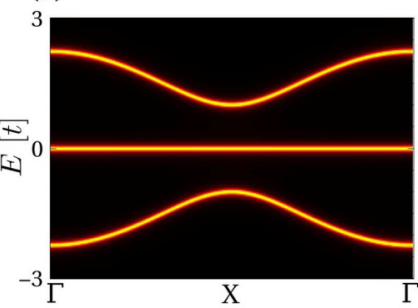

$\mathrm{X}$
FIG. 1. (a) Sketch of a Kondo lattice coupled to a flat-band electron gas. Panels [(b), (c)] show the lattice model realizing a flatband Kondo lattice model in two dimension (b) and one dimension (c). Panels [(d), (e)] show the momentum-resolved spectral function $A(\mathbf{k}, \omega)$ of the flat-band electron gas of panels [(b), (c)], respectively. 
the conduction bath. However, in the flat-band limit, the previous picture breaks down due to the absence of a well-defined Fermi surface.

Here we address the fate of a flat-band system coupled to a lattice of magnetic impurities, realizing the so-called flat-band Kondo lattice model. We observe that the system develops a robust local magnetic order, overcoming Kondo screening effects of the flat band. We demonstrate that the full phenomenology can be captured by symmetry broken mean-field method and compare these results with two genuine manybody methods, dynamical mean-field theory (DMFT) and tensor networks states. Our results demonstrate the nontrivial impact of flat bands in Kondo lattice problems, emphasizing the complex interplay between exchange and Kondo physics in flat-band systems.

The paper is organized as follows. In Section II, we introduce the minimal models featuring a flat-band Kondo lattice physics, both in one dimension and in two dimensions. In Section III we present the solution of the two-dimensional flatband Kondo lattice model as solved by DMFT. In Section IV we present the solution of the one-dimensional flat-band Kondo lattice model as solved with tensor networks. Finally, in Section $\mathrm{V}$ we summarize our conclusions.

\section{FLAT-BAND KONDO LATTICE MODEL}

In the following we describe the effective models used to capture a flat-band Kondo lattice model, as shown in Figs. 1(b) and $1(\mathrm{c})$. The full Hamiltonian $\mathcal{H}=\mathcal{H}_{t}+\mathcal{H}_{\text {int }}$ has both noninteracting $\mathcal{H}_{t}$ and interacting $\mathcal{H}_{\text {int }}$ terms. Let us start with the noninteracting term, for which we take a first-neighbor spinful model of the form

$$
\mathcal{H}_{t}=t \sum_{\langle i j\rangle, \sigma} c_{i \sigma}^{\dagger} c_{j \sigma},
$$

where $c_{i \sigma}$ is the annihilation operator for site $i$ and spin $\sigma$, and \langle\rangle denotes nearest-neighbor sites. The lattices considered for Eq. (1) would be a square lattice and a ladder for the twodimensional and one-dimensional cases, respectively. In both cases, the fundamental unit cell of the system consists of four spinful sites.

Let us now address the interacting term of the Hamiltonian. In each unit cell of the system, we will include interactions solely in a single site, which we label by $I$ in the form

$$
\mathcal{H}_{\text {int }}=U \sum_{I}\left(n_{I \uparrow}-\frac{1}{2}\right)\left(n_{I \downarrow}-\frac{1}{2}\right),
$$

where $n_{I \sigma}=c_{I \sigma}^{\dagger} c_{I \sigma}$ and $U>0$ is the interaction strength. The interaction acts only at one of every four sites, namely, the sites labeled as $I$, which in the following will be referred to as the impurity site. It is worth noting that by definition, the previous Hamiltonian leads to a half filled state for arbitrary $U$. In the strong coupling limit $U \gg t$, the low-energy sector realizes a flat-band electron gas, as shown in Figs. 1(d) and 1(e). The interacting site develops a local magnetic moment, leading to a low-energy effective Hamiltonian (Appendix A) for the full system of the form

$$
\mathcal{H}_{\mathrm{eff}}=\sum_{\mathbf{k}, \sigma} \psi_{\mathbf{k} \sigma}^{\dagger} h_{t}(\mathbf{k}) \psi_{\mathbf{k} \sigma}+J \sum_{\mathbf{k} \mathbf{k}^{\prime}} \mathbf{S}_{\mathbf{I}} \cdot \tau_{\sigma, \sigma^{\prime}} \psi_{\mathbf{k}, \sigma}^{\dagger} \psi_{\mathbf{k}^{\prime}, \sigma^{\prime}},
$$

where $\psi_{\mathbf{k} \sigma}=\left(\begin{array}{lll}c_{A \sigma} & c_{B \sigma} & c_{C \sigma}\end{array}\right)^{T}$ and $h_{t}(\mathbf{k})$ is the superlattice Fourier transform of the hopping matrix of noninteracting sites. and $J=t^{2} / U$ is the effective Kondo coupling. In the following we will work with the full fermionic Hamiltonian $\mathcal{H}$, yet the effective Hamiltonian as shown in Eq. (3) will provide useful insights to rationalize the full many-body solution.

Finally, it is worth commenting on the emergence of the flat band in the previous models. In the infinite $U$ limit, one of the four sites is fully disconnected from the other noninteracting sites. In this limit, the two-dimensional and one-dimensional models realize a bipartite lattice with a different number of sublattice sites per unit cell, automatically leading to a flat band [52-55]. For a finite $U$, the system will thus realize a two-dimensional or one-dimensional electron gas coupled to a lattice of Kondo impurities. In the following, we explore this finite $U$ coupling limit, first in two dimensions with DMFT and later in one dimension with tensor networks.

Originally, the Kondo lattice model $[34,56]$ was proposed to account for the competition between the Kondo effect and magnetic order in various heavy fermion alloys. At very low temperatures, the localized impurity spin is completely screened by the dispersive conduction electrons via onsite Kondo exchange interaction, leading to the formation of a coherent Kondo spin-singlet heavy fermion state. Besides, the local coupling between impurity spins and conduction electrons may give rise to a magnetic order through the intersite RKKY interaction [49-51]. Doniach [57] proposed a phase diagram with a quantum phase transition between two distinct phases, i.e., a magnetically ordered one and a nonmagnetic Kondo phase. Here we discuss a different class of heavy fermion system where the coexistence between the magnetic order and the Kondo behavior is due to partial screening of the impurity by conduction electrons, which has been observed [58,59] experimentally.

Kondo lattice Hamiltonian has been studied previously in the context of a partially screened magnetically ordered state for conventional dispersive conduction electrons using mean-field theory [60], DMFT [61,62], quantum Monte Carlo simulation [63], and variational Monte Carlo [64]. Coexistence of the partially magnetically ordered state along with the Kondo screened phase has been attributed to frustrated lattice geometry [64], long-range electron hopping [65], site-selective Kondo screening [66], competition of Kondo screening, and RKKY interaction in the low density of conduction electron [60]. The full fermionic Anderson model displays richer physics than its counterpart Kondo lattice model, as it allows the charge fluctuation at the impurity site as well. Here we study the nature of such an underscreened magnetically ordered phase with the conduction band having a flat(quasiflat) band. Such an underscreened phase is stable at finite temperature and local exchange field.

\section{DMFT APPROACH OF THE TWO-DIMENSIONAL FLAT-BAND KONDO LATTICE MODEL}

In the following, we will address the fate of the twodimensional Kondo lattice problem. We will consider the mean-field solution as a reference to see how quantum fluctuations included in the dynamical mean-field approach renormalize the results. Evaluations of the site-selective 
magnetism have been calculated using the cellular mean-field theory, cellular DMFT with continuous time quantum Monte Carlo method, and exact diagonalization (ED) as the impurity solver [67-69]. Within CDMFT, a lattice problem is mapped to a finite cluster coupled to a noninteracting bath. In our case, the cluster is a four-site $(2 \times 2)$ plaquette as shown in Fig. 1 [70-72]. We define the site-dependent absolute magnetization for the cluster as $m_{\mathrm{i}}=\left\langle n_{i \uparrow}\right\rangle-\left\langle n_{i \downarrow}\right\rangle$, where $\left\langle n_{i, \sigma}\right\rangle=$ $G_{i, \sigma \sigma}\left(\tau \rightarrow 0^{-}\right)$is the density of spin- $\sigma$ particles for a given site of the cluster calculated from the local Green's function. To understand the origin of the site-selective magnetic order, we calculate the effective hybridization [73] between the impurity and the sites near it. We can define the nonlocal effective hybridization order for the given four-site clusters as

$$
\Delta_{\alpha}=\left|\sum_{\alpha \neq \beta, \sigma}\left\langle c_{\alpha \sigma}^{\dagger} c_{\beta \sigma}\right\rangle\right|=\sum_{\alpha \neq \beta, \sigma}\left|G_{i, \sigma \sigma}^{\alpha \beta}\left(\tau \rightarrow 0^{-}\right)\right|,
$$

where $\alpha(\beta)$ is the sublattice index. The behavior of effective hybridization is proportional to the noninteracting local density of states (LDOS) at the sublattices of the unit cell [35]. In the following, we present the complete magnetic phase diagram in the presence of a finite two-body interaction $U$ at finite temperature $T$ at half filling, where the number of particles per site is one. Simultaneously, we also explore the nature of effective Kondo hybridization on the emergent site-selective magnetization. The role of quantum fluctuations, ignored in mean-field theory, has been addressed by using DMFT.

\section{A. Zero-temperature calculations}

Due to emergent singularity in the LDOS at different sublattices in the presence of the finite local interaction at the impurity site, the local magnetic order is nonuniform across different sites. We show the spatially resolved magnetic order $m_{\alpha}$ evaluated using Hartree-Fock mean-field theory in Fig. 2(a), zero-temperature ED+CDMFT in Fig. 2(b), and continuous-time quantum Monte Carlo (CTQMC) +CDMFT at $T=0.01$ in Fig. 2(c) for varying interaction strength $U / t$ at the impurity site. We allow the breaking of the $S U(2)$ spin-rotation symmetry to capture the magnetically ordered state. An initial self-energy that is constant in the Matsubara frequency is added in this way that it breaks $\mathrm{SU}(2)$ symmetry of the Hamiltonian.

For weak to moderate interactions, the local magnetic order at different sublattices gradually develops such that $\operatorname{sign}\left(m_{A / B}\right)=-\operatorname{sign}\left(m_{C}\right)=-\operatorname{sign}\left(m_{I}\right)$ for any $U / t>0$ due to the antiferromagnetic coupling between the local moments at a neighboring site of the unit cell. The sizes of the magnetic moments of the $A / B, C$, and $I$ sites are very different, as expected from the nonuniform nature of the model. While the magnetization of the $A / B$ and $I$ sites saturates with the strength of the interaction, the same does not occur on site $C$. Magnetic order at the $C$ site asymptotically goes to zero after attaining the peak at a given $U / t$. The qualitative behavior obtained from the different approaches is similar for all $U / t$; however, the key aspect is the amplitude of $m_{\alpha}$ calculated using DMFT that is significantly smaller than the one obtained from MF approach. This is due to the many-body correc-
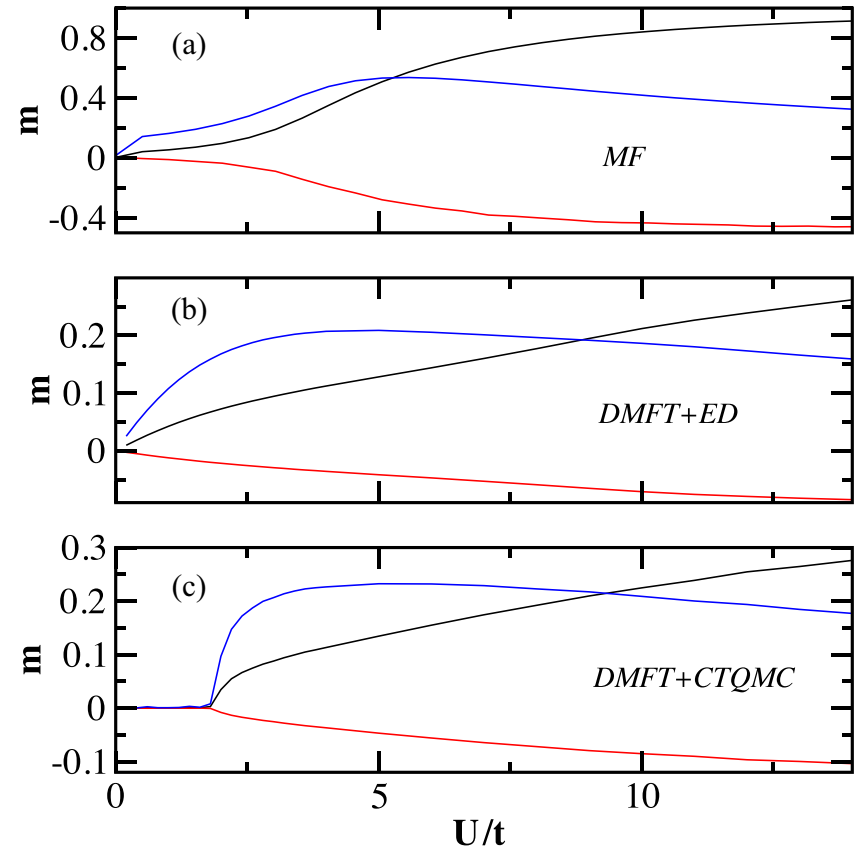

FIG. 2. Magnetic order parameter at impurity site, $I$ (black), at sites next to the impurity, $A / B$ (red), and at the second-neighbor site of the impurity site, $C$ (blue), evaluated using (a) mean-field theory [74]. (b) DMFT + ED and (c) DMFT + CTQMC for varying interaction strength $U$. Note that the magnitude of the magnetic order at $C$ site has been scaled by a factor of four for the purpose of visual clarity.

tions included in DMFT not accounted for by the mean-field calculations. Interestingly, and despite such differences, it is observed that the flat-band electron gas does not fully screen the local moment. The hybridization between the electrons at the noninteracting sites and the impurity site leads to the formation of the singlet between the spins of electrons at different sites. In contrast, the RKKY interaction makes the impurity spins interacting with each other via conduction electrons and thus tends to stabilize the magnetic ordering. This can be shown by the finite hybridization along with the local magnetic order at $U / t$ (Fig. 3). Effective hybridization decreases monotonically both at the impurity sites and the site next to the impurity such that $\Delta_{I}<\Delta_{A / B}$ for all $U / t$, consistent with the observed magnetic order at the corresponding site. Effective hybridization at the second-neighbor site decreases initially and then increases further, congruous with magnetic order.

To understand the interplay of the Kondo screening and the RKKY interaction, we show the evolution of the mean-field LDOS at the impurity site and its vicinity in Fig. 4 for varying $U$. For $U \gg 1$, the local DOS at the second-neighbor site vanishes at the Fermi energy like the Dirac electron pseudogap, whereas the nearest neighbor to the impurity site exhibits a flat band. In particular, in the infinite $U$ limit, the impurity sites are fully disconnected from the other noninteracting sites. In such case, the system becomes an array of Kondo impurities in a bath of noninteracting electrons. The resultant geometry is analogous to the array of Kondo impurity on the Lieb lattice. The impurity hybridizes with the noninteracting bath via $A$ and $B$ sites. The Lieb lattice is known to give rise to spatially localized electronic flat bands $[55,75]$. The origin of 


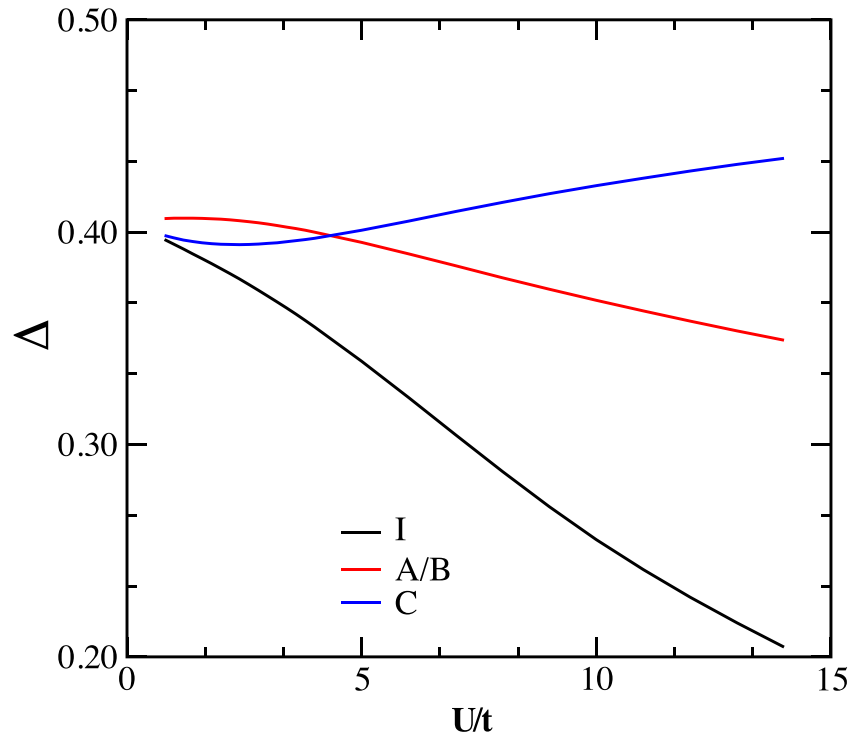

FIG. 3. Effective hybridization of a given sublattice with the rest of the sites in the unit cell as Eq. (4).

the flat band is due to the destructive interference of the wave functions $[55,76]$.

In general, the Kondo effect describes the screening of the spatially localized impurity by the delocalized bath of noninteracting electrons [35]. The nature of the screening is antiferromagnetic in nature. Localization of the bath electrons makes them less available for screening the localized impurities and thus making the impurities underscreened. As evident from the local DOS (Fig. 4), noninteracting conduction electrons get localized at the site $A(B)$ for $U \gg t$ and thus partially screening the impurity at the $I$ site. The Kondo effect does

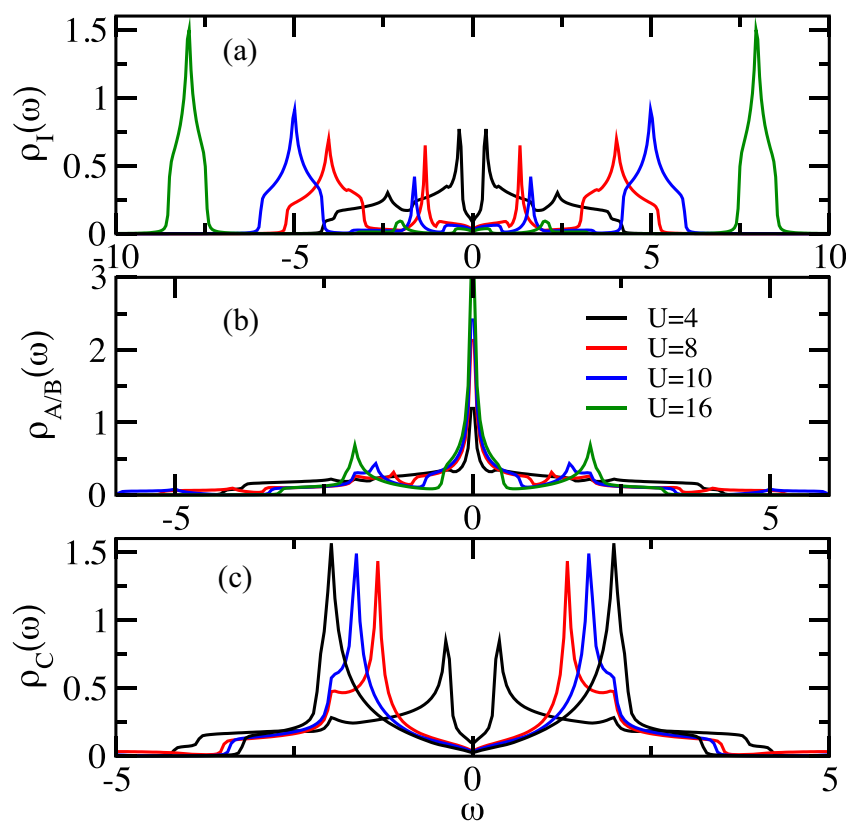

FIG. 4. Evolution of the mean-field LDOS at (a) the impurity site, (b) at sites next to the impurity, and (c) at a second-neighbor site on the impurity site.
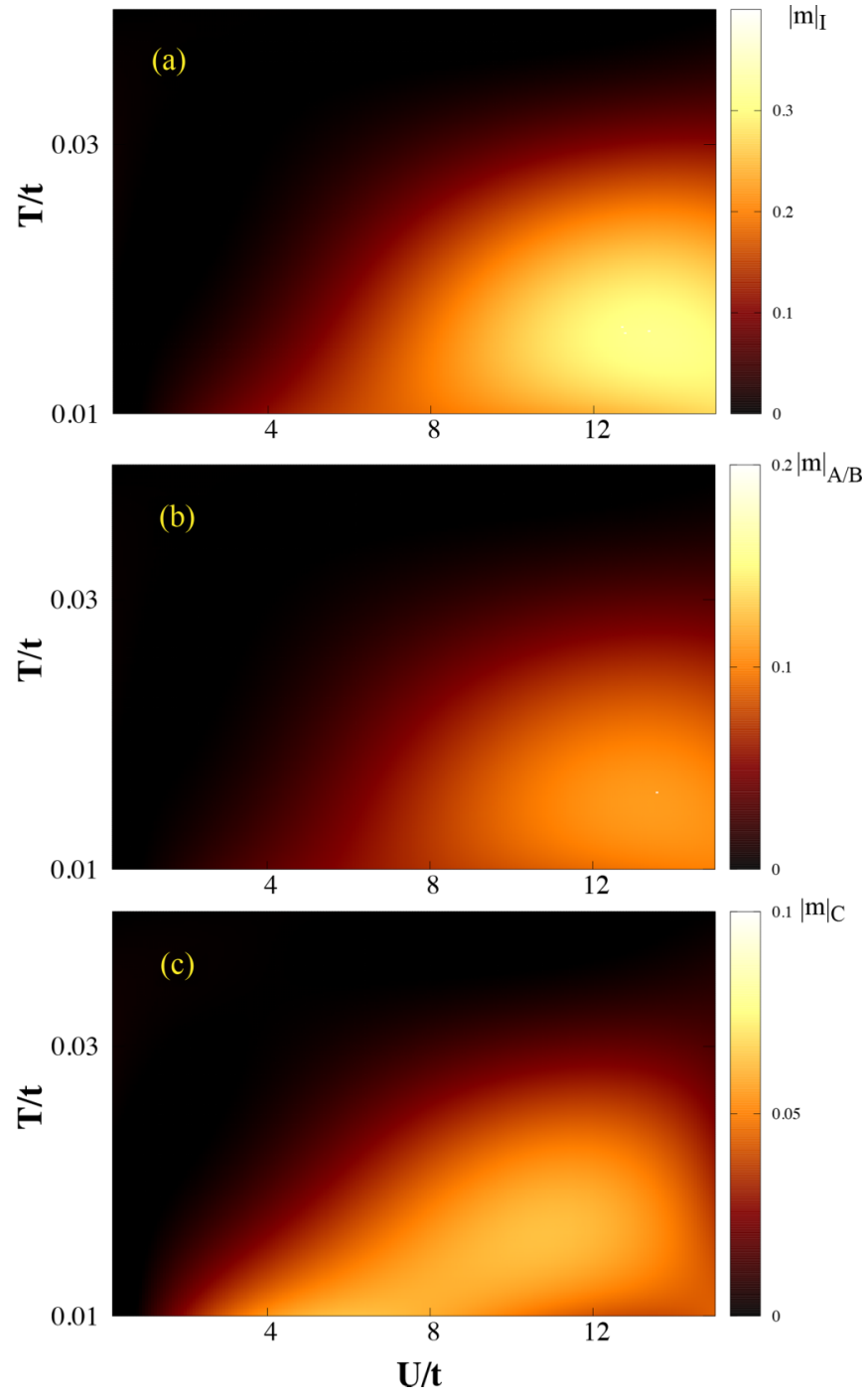

FIG. 5. Temperature vs interaction strength phase diagram of the inhomogeneous Hubbard model showing local magnetization at (a) impurity site, $m_{\mathrm{I}}$; (b) at site next to the impurity, $m_{\mathrm{A} / \mathrm{B}}$; and (c) second-neighbor site to the impurity site, $m_{\mathrm{C}}$.

not lead to a complete screening of the localized spins, and the exchange between the (underscreened) spins leads to the formation of the magnetic order. The coexistence between magnetic order and the Kondo effect can be attributed to the presence of the flat bands.

\section{B. Finite temperature calculations}

We now move on to consider the effects of finite temperature in the DMF calculations. First, it is worth noting that the current model will not show long-range magnetic order due to its two-dimensional nature. DMFT, however, does not capture long-range fluctuations and therefore does not account for the Mermin-Wagner theorem [77,78]. Within this framework, the existence of a finite magnetization in a DMFT calculation must be rationalized as a signature of strong magnetic correlation, as addressed in other systems [79]. In this context, while the true long-range ordering temperature is at $T=0$, correlation lengths start to grow at a specific temper- 

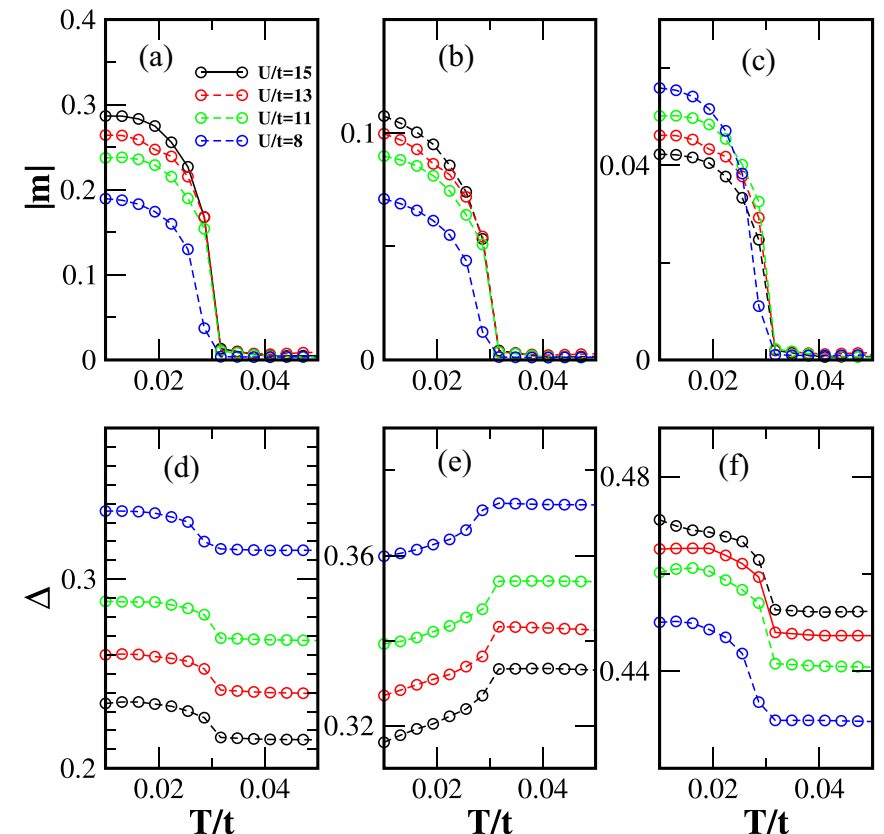

FIG. 6. Local magnetic order [(a)-(c)] and the corresponding effective hybridization [(d)-(f)] for the impurity site I, site next to impurity site $\mathrm{A} / \mathrm{B}$, and second-neighbor site $\mathrm{C}$ of the impurity with varying temperature.

ature scale $T^{*}>0$ and is the temperature scale captured by DMFT [79].

In Fig. 5, we show the region in the T-U plane where the magnetic order, $m_{\alpha}$, is finite. The amplitude of $m_{\alpha}$, at a given $U / t$, is site dependent. Local magnetization at the impurity site and at the site next to the impurity monotonically increases and then saturates with increasing interaction strength with a nonmagnetic to magnetic transition at $T_{c}$. However, the magnetic behavior at the second-neighbor site gets peaked at $U_{p} / t$ for a given $T$. $U_{p}$ increases monotonically with the $T$. We show the spatially resolved magnetic order $m_{\alpha}$ the corresponding effective hybridization evaluated using CTQMC+CDMFT with varying temperatures at different interaction strength $U / t$ in the upper panels and lower panels in Fig. 6, respectively. As the temperature is increased, the magnetic order for all sublattices sharply disappears at $T_{c}$. The evaluation of the magnetic order at the second-neighbor site decreases with increasing value of $U$ for a given $T$, opposite to the impurity site and the site next to the impurity. Effective hybridization changes with the transition temperature, with a cusp at $T_{c}$. It is important to note that the effective hybridization is finite in both magnetic and nonmagnetic regions, and the behavior of $\Delta$ is consistent with the corresponding local magnetic order.

\section{TENSOR NETWORK APPROACH TO THE ONE-DIMENSIONAL FLAT-BAND KONDO LATTICE MODEL}

We now move on to consider a one-dimensional flat-band Kondo lattice model, which can be rationalized as a onedimensional version of the model outlined above. First, it is worth emphasizing that for a one-dimensional quantum many-

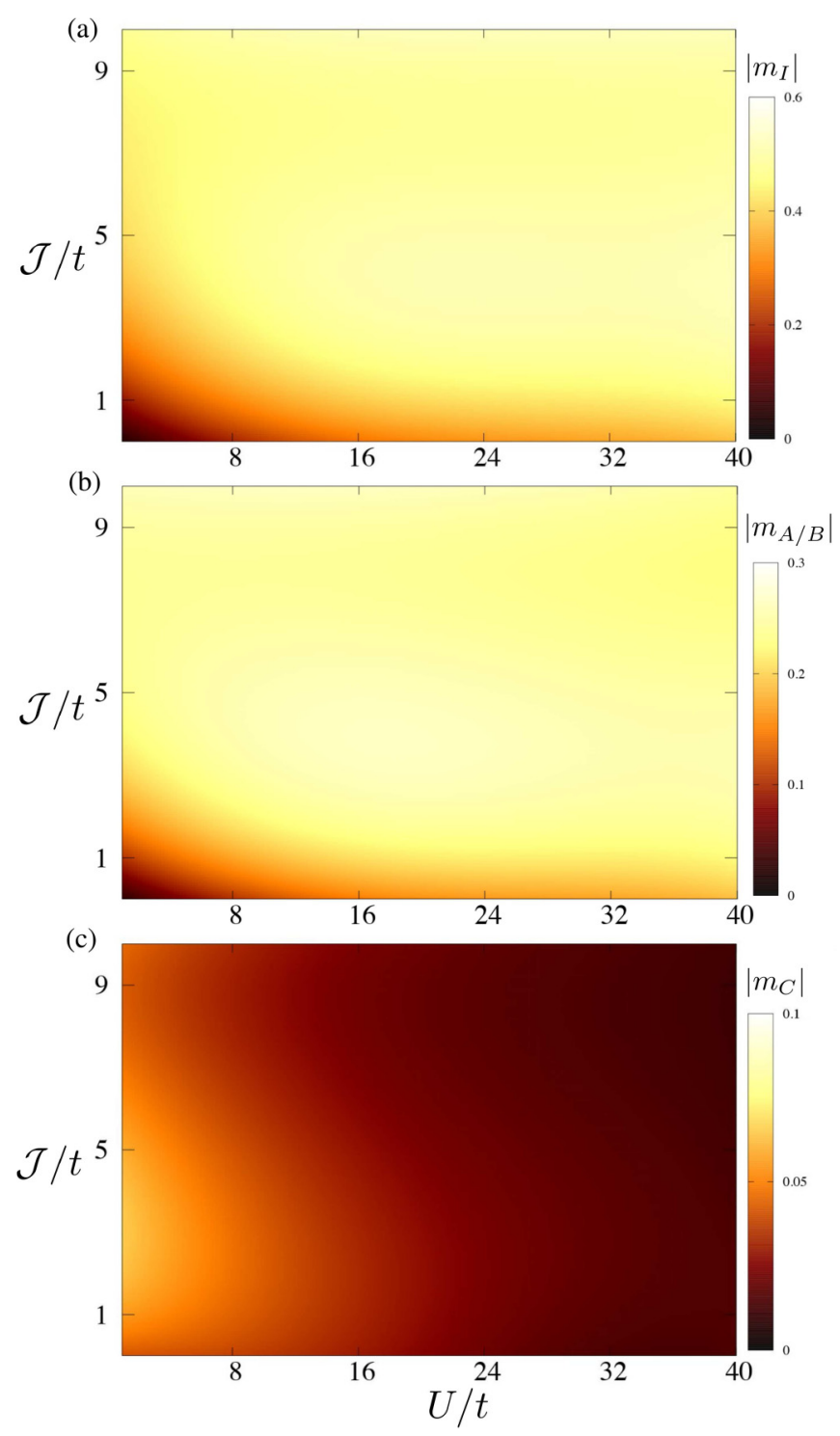

FIG. 7. Local magnetic order on (a) impurity site I, (b) flat-band sites $\mathrm{A}$ and $\mathrm{B}$, and (c) site $\mathrm{C}$ vs Hubbard $\mathrm{U}$ and local exchange field $\mathcal{J} / t$ for the generalized one-dimensional model Eq. (5).

body problem, the existence of strong quantum many-body fluctuations would prevent the system to order even in the presence of strong magnetic correlations. To accommodate this restriction, we will generalize the flat-band Kondo lattice model, including local symmetry-breaking terms, allowing for a selective quench of the quantum fluctuations of the system.

Let us now comment on the specific model we will address. On the computational side, the interacting model can be effectively solved using tensor network methods [80-83] due to its one-dimensional nature. We will include a noninteracting hopping term in the geometry shown in Fig. 1(c) and whose flat-band structure is shown in Fig. 1(f). Local Hubbard interactions are included in site $I$ as depicted in Fig. 1(d). On top of the interacting term, we will also include a local exchange field on the impurity site, leading to a full Hamiltonian of the 


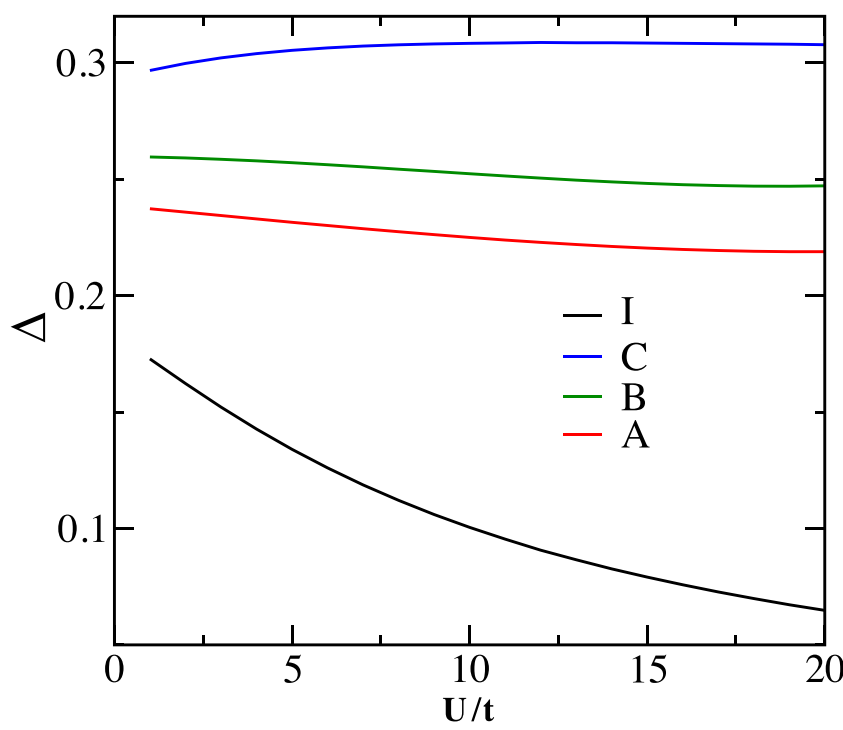

FIG. 8. Effective hybridization at different sublattice sites in the one-dimensional flat-band Kondo lattice model with $\mathcal{J}=5 t$ for varying $U / t$.

form

$$
\mathcal{H}=t \sum_{\langle i, j\rangle} c_{i}^{\dagger} c_{j}+U \sum_{i \in I}\left(n_{i \uparrow}-\frac{1}{2}\right)\left(n_{i \downarrow}-\frac{1}{2}\right)+\mathcal{J} \sum_{I} S_{i}^{z}
$$

Increasing the local exchange $\mathcal{J}$ can lead to the emergence of the quasiflat band at the site next to the impurity site, even at $U=0$. In a mean-field language, $\mathcal{J}$ could be rationalized as an effective local magnetization, and therefore $\mathcal{J}$ allows to effectively interpolate between a mean-field and purely manybody limit. In particular, when $\mathcal{J} \rightarrow \infty$, the impurity site is fully magnetized, and we are in pure mean-field limit, whereas when $\mathcal{J}=0$, the magnetic instability solely originates from $U$ and we are in a pure many-body limit. To be concrete, we consider a chain (Fig. 7) of 40 sites (i.e., 20 lattice constants long) and compute averaged magnetization on the impurity sites $I$, the flat-band sites $A$ and $B$, and the next-nearest site $\mathrm{C}$ with varying Hubbard $U$ and the field $\mathcal{J}$ of impurity sites. We find that the local exchange $\mathcal{J}$ destroys quantum fluctuations and promotes a quasi-long-range order at the impurity site and sites $A / B$. As $\mathcal{J}$ becomes smaller, larger $U$ is required to produce the magnetic order.

The above behavior is consistent with the hybridization effects in the two-dimensional flat-band Kondo lattice addressed previously with DMFT. In particular, we show the effective hybridization varying with $U$ at fixed field $\mathcal{J}=5 t$ in Fig. 8. We find that the effective hybridization at all sublattice sites has the same behavior as in the two-dimensional case in Fig. 3. Due to the finite exchange field $\mathcal{J}$, the hybridization at all sites is suppressed, with the impurity site having the largest suppression. The previous results show that, while the one-dimensional model is formally different from its two-dimensional counterpart, magnetic correlations between Kondo sites and flat-band sites show a similar phenomenology.

\section{CONCLUSION}

Kondo lattice problems represent one of the paradigmatic many-body problems, hosting a variety of intricate phenomena due to the competition of Kondo screening and magnetic ordering. Here we have addressed the physics of a Kondo lattice problem, in which a conventional dispersive electron gas is replaced by a flat-band electronic state. In particular, we considered flat-band Kondo lattice models in both two and one dimensions, which we solved using many-body DMFT and tensor networks, respectively. We have demonstrated the emergence of a locally ordered state, showing how the formation of the Kondo cloud is overcome by the magnetic correlation between impurities. Interestingly, the underscreened phase observed in our many-body results can be captured via a conventional symmetry-broken mean-field method, highlighting how the main features of the system are qualitatively reproduced. Our treatment focused purely on the half-filled case, yet doping of the Kondo lattice is expected to lead to a more complex interplay of the exchange and Kondo coupling scales, therefore leading to substantially more complex phase diagrams. Our results exemplify the fine interplay between magnetic ordering and many-body screening, putting forward flat-band Kondo lattice models as a powerful platform to explore exotic emergent quantum many-body states.

\section{ACKNOWLEDGMENTS}

We acknowledge the computational resources provided by the Aalto Science-IT project. J.L.L. acknowledges financial support from the Academy of Finland Projects No. 331342 and No. 336243, and the Jane and Aatos Erkko Foundation. We thank A. Ramires, P. Törmä, P. Liljeroth, and M. Aapro for useful discussions.

\section{APPENDIX: LOW-ENERGY EFFECTIVE KONDO LATTICE MODEL}

The tight-binding full fermionic Hamiltonian in momentum space can be written as $\mathcal{H}=\mathcal{H}_{0}+\mathcal{H}_{V}$ such that

$$
\begin{gathered}
\mathcal{H}_{0}=\sum_{\mathbf{k} \sigma} \psi_{\mathbf{k} \sigma}^{\dagger} h_{t}(\mathbf{k}) \psi_{\mathbf{k} \sigma}+\mathcal{H}_{i n t} \\
\mathcal{H}_{V}=\sum_{\mathbf{k} \sigma}\left(\psi_{\mathbf{k} \sigma}^{\dagger} V_{t}(\mathbf{k}) \psi_{I \sigma}+\text { H.c. }\right),
\end{gathered}
$$

where $\psi_{I \sigma}=\left(c_{I \sigma}, 0,0\right)^{T}$. Schrieffer-Wolff (SW) transformation can be used to get the low-energy effective Kondo lattice model. SW transformation Anderson Hamiltonian with empty, singly and doubly occupied impurity states to Kondo lattice Hamiltonian with singly occupied state. SW transformation in general can be written as

$$
\mathcal{H}_{\text {eff }}=e^{-S} \mathcal{H} e^{S}=\mathcal{H}+\frac{1}{2}[S, \mathcal{H}]+\frac{1}{3}[S,[S, \mathcal{H}]]+.
$$

$\mathrm{S}$ is chosen in such a way that it cancels the off-diagonal part, i.e., hybridization of the impurity with noninteracting bath to the first order, so that

$$
\mathcal{H}_{V}+\left[S, \mathcal{H}_{V}\right]=0
$$

and the low-energy effective Hamiltonian become

$$
\mathcal{H}_{\text {eff }}=\mathcal{H}_{0}+\frac{1}{2}\left[S, \mathcal{H}_{V}\right]
$$


An explicit form of $S$ for can be constructed as

$$
S=\sum_{\mathbf{k} j \sigma}\left\{A_{\mathbf{k}}+B_{\mathbf{k}} n_{I \bar{\sigma}}\right\} V_{t}(\mathbf{k})\left(\psi_{\mathbf{k} \sigma}^{\dagger} \psi_{I \sigma}-\text { H.c. }\right),
$$

where

$$
A=\frac{1}{h_{t}(\mathbf{k})} B=\frac{1}{h_{t}(\mathbf{k})-U}-\frac{1}{h_{t}(\mathbf{k})} .
$$

Using Eq. (A6) in the Eq. (A5), we write effective Kondo lattice Hamiltonian given as

$$
\mathcal{H}_{\mathrm{eff}}=\sum_{\mathbf{k}, \sigma} \psi_{\mathbf{k} \sigma}^{\dagger} h_{t}(\mathbf{k}) \psi_{\mathbf{k} \sigma}+\sum_{\mathbf{k} \mathbf{k}^{\prime} \sigma \sigma^{\prime}} J_{\mathbf{k}, \mathbf{k}^{\prime}} \mathbf{S}_{\mathbf{I}} \cdot \tau_{\sigma, \sigma^{\prime}} \psi_{\mathbf{k}, \sigma}^{\dagger} \psi_{\mathbf{k}^{\prime}, \sigma^{\prime}},
$$

with the exchange coupling as

$$
\begin{aligned}
J_{\mathbf{k} \mathbf{k}^{\prime}}= & V_{t}(\mathbf{k}) V_{t}^{*}\left(\mathbf{k}^{\prime}\right)\left\{-\left(h_{t}(\mathbf{k})-U\right)^{-1}\right. \\
& \left.-\left(h_{t}\left(\mathbf{k}^{\prime}\right)-U\right)^{-1}+h_{t}(\mathbf{k})^{-1}+h_{t}\left(\mathbf{k}^{\prime}\right)^{-1}\right\},
\end{aligned}
$$

where $V_{t}(\mathbf{k})$ is the super lattice Fourier transform of the hopping matrix of noninteracting sites and the impurity. For strong coupling, We can consider $V(\mathbf{k})=V(\mathbf{k})=t$ and and conduction band energies close to the Fermi energy can be neglected. Thus, the simplified form of the exchange coupling can be written as $t^{2} / U$.
[1] J. Ruostekoski, Optical Kagome Lattice for Ultracold Atoms with Nearest Neighbor Interactions, Phys. Rev. Lett. 103, 080406 (2009).

[2] V. I. Iglovikov, F. Hébert, B. Grémaud, G. G. Batrouni, and R. T. Scalettar, Superconducting transitions in flat-band systems, Phys. Rev. B 90, 094506 (2014).

[3] N. B. Kopnin, T. T. Heikkilä, and G. E. Volovik, Hightemperature surface superconductivity in topological flat-band systems, Phys. Rev. B 83, 220503 (2011).

[4] M. Tovmasyan, S. Peotta, P. Törmä, and S. D. Huber, Effective theory and emergent SU(2) symmetry in the flat bands of attractive hubbard models, Phys. Rev. B 94, 245149 (2016).

[5] J. S. Hofmann, E. Berg, and D. Chowdhury, Superconductivity, pseudogap, and phase separation in topological flat bands, Phys. Rev. B 102, 201112(R) (2020).

[6] V. Peri, Z.-D. Song, B. A. Bernevig, and S. D. Huber, Fragile Topology and Flat-Band Superconductivity in the StrongCoupling Regime, Phys. Rev. Lett. 126, 027002 (2021).

[7] R. Drost, T. Ojanen, A. Harju, and P. Liljeroth, Topological states in engineered atomic lattices, Nat. Phys. 13, 668 (2017).

[8] M. R. Slot, T. S. Gardenier, P. H. Jacobse, G. C. P. van Miert, S. N. Kempkes, S. J. M. Zevenhuizen, C. M. Smith, D. Vanmaekelbergh, and I. Swart, Experimental realization and characterization of an electronic lieb lattice, Nat. Phys. 13, 672 (2017).

[9] M. N. Huda, S. Kezilebieke, and P. Liljeroth, Designer flat bands in quasi-one-dimensional atomic lattices, Phys. Rev. Research 2, 043426 (2020).

[10] A. A. Khajetoorians, D. Wegner, A. F. Otte, and I. Swart, Creating designer quantum states of matter atom-by-atom, Nat. Rev. Phys. 1, 703 (2019).

[11] G.-B. Jo, J. Guzman, C. K. Thomas, P. Hosur, A. Vishwanath, and D. M. Stamper-Kurn, Ultracold Atoms in a Tunable Optical Kagome Lattice, Phys. Rev. Lett. 108, 045305 (2012).

[12] S. Taie, H. Ozawa, T. Ichinose, T. Nishio, S. Nakajima, and Y. Takahashi, Coherent driving and freezing of bosonic matter wave in an optical lieb lattice, Sci. Adv. 1, e1500854 (2015).

[13] I. Bloch, J. Dalibard, and S. Nascimbène, Quantum simulations with ultracold quantum gases, Nat. Phys. 8, 267 (2012).

[14] E. Suárez Morell, J. D. Correa, P. Vargas, M. Pacheco, and Z. Barticevic, Flat bands in slightly twisted bilayer graphene: Tight-binding calculations, Phys. Rev. B 82, 121407(R) (2010).

[15] Y. Cao, V. Fatemi, S. Fang, K. Watanabe, T. Taniguchi, E. Kaxiras, and P. Jarillo-Herrero, Unconventional superconduc- tivity in magic-angle graphene superlattices, Nature (London) 556, 43 (2018).

[16] E. Y. Andrei, D. K. Efetov, P. Jarillo-Herrero, A. H. MacDonald, K. F. Mak, T. Senthil, E. Tutuc, A. Yazdani, and A. F. Young, The marvels of moiré materials, Nat. Rev. Mater. 6, 201 (2021).

[17] D. Leykam, A. Andreanov, and S. Flach, Artificial flat band systems: from lattice models to experiments, Adv. Phys.: X 3, 1473052 (2018).

[18] T. T. Heikkilä, N. B. Kopnin, and G. E. Volovik, Flat bands in topological media, JETP Lett. 94, 233 (2011).

[19] O. Derzhko, J. Richter, and M. Maksymenko, Strongly correlated flat-band systems: The route from heisenberg spins to hubbard electrons, Int. J. Mod. Phys. B 29, 1530007 (2015).

[20] S. Peotta and P. Törmä, Superfluidity in topologically nontrivial flat bands, Nat. Commun. 6, 8944 (2015).

[21] K. Noda, K. Inaba, and M. Yamashita, Flat-band ferromagnetism in the multilayer lieb optical lattice, Phys. Rev. A 90, 043624 (2014).

[22] N. Swain and M. Karmakar, Strain-induced superconductorinsulator transition on a lieb lattice, Phys. Rev. Research 2, 023136 (2020).

[23] Y. Cao, V. Fatemi, A. Demir, S. Fang, S. L. Tomarken, J. Y Luo, J. D. Sanchez-Yamagishi, K. Watanabe, T. Taniguchi, E. Kaxiras, R. C. Ashoori, and P. Jarillo-Herrero, Correlated insulator behaviour at half-filling in magic-angle graphene superlattices, Nature (London) 556, 80 EP (2018).

[24] M. Yankowitz, S. Chen, H. Polshyn, Y. Zhang, K. Watanabe, T. Taniguchi, D. Graf, A. F. Young, and C. R. Dean, Tuning superconductivity in twisted bilayer graphene, Science 363, 1059 (2019).

[25] M.-T. Tran and T. T. Nguyen, Molecular kondo effect in flatband lattices, Phys. Rev. B 97, 155125 (2018).

[26] A. Ramires and J. L. Lado, Emulating Heavy Fermions in Twisted Trilayer Graphene, Phys. Rev. Lett. 127, 026401 (2021).

[27] Y. Jiang, X. Lai, K. Watanabe, T. Taniguchi, K. Haule, J. Mao, and E. Y. Andrei, Charge order and broken rotational symmetry in magic-angle twisted bilayer graphene, Nature (London) 573, 91 (2019).

[28] X. Y. Xu, K. T. Law, and P. A. Lee, Kekulé valence bond order in an extended hubbard model on the honeycomb lattice with possible applications to twisted bilayer graphene, Phys. Rev. B 98, 121406(R) (2018). 
[29] P. Kumar, T. I. Vanhala, and P. Törmä, Magnetization, $d$ wave superconductivity, and non-Fermi-liquid behavior in a crossover from dispersive to flat bands, Phys. Rev. B 100, 125141 (2019).

[30] P. Kumar, S. Peotta, Y. Takasu, Y. Takahashi, and P. Törmä, Flat-band-induced non-fermi-liquid behavior of multicomponent fermions, Phys. Rev. A 103, L031301 (2021).

[31] P. Coleman, New approach to the mixed-valence problem, Phys. Rev. B 29, 3035 (1984).

[32] G. R. Stewart, Non-fermi-liquid behavior in $d$ - and $f$-electron metals, Rev. Mod. Phys. 73, 797 (2001).

[33] S. Wirth and F. Steglich, Detaching the antiferromagnetic quantum critical point from the Fermi-surface reconstruction in $\mathrm{YbRh}_{2} \mathrm{Si}_{2}$, Nat. Phys. 5, 465 (2009).

[34] H. Tsunetsugu, M. Sigrist, and K. Ueda, The ground-state phase diagram of the one-dimensional Kondo lattice model, Rev. Mod. Phys. 69, 809 (1997).

[35] Q. Si and F. Steglich, Heavy fermions and quantum phase transitions, Science 329, 1161 (2010).

[36] P. Coleman, Heavy fermions and the Kondo lattice: a 21 st century perspective, arXiv:1509.05769 (2015).

[37] A. Ramires and P. Coleman, Theory of the Electron Spin Resonance in the Heavy Fermion Metal $\beta-\mathrm{YbAlB}_{4}$, Phys. Rev. Lett. 112, 116405 (2014).

[38] A. Ramires and P. Coleman, Supersymmetric approach to heavy fermion systems, Phys. Rev. B 93, 035120 (2016).

[39] V. Vaňo, M. Amini, S. C. Ganguli, G. Chen, J. L. Lado, S. Kezilebieke, and P. Liljeroth, Artificial heavy fermions in a van der Waals heterostructure, arXiv:2103.11989 [cond-mat.meshall].

[40] M. Dzero, K. Sun, V. Galitski, and P. Coleman, Topological Kondo Insulators, Phys. Rev. Lett. 104, 106408 (2010).

[41] M. Dzero, J. Xia, V. Galitski, and P. Coleman, Topological Kondo insulators, Annu. Rev. Condens. Matter Phys. 7, 249 (2016).

[42] M. Legner, A. Rüegg, and M. Sigrist, Surface-State Spin Textures and Mirror Chern Numbers in Topological Kondo Insulators, Phys. Rev. Lett. 115, 156405 (2015).

[43] L. Jiao, S. Howard, S. Ran, Z. Wang, J. O. Rodriguez, M. Sigrist, Z. Wang, N. P. Butch, and V. Madhavan, Chiral superconductivity in heavy-fermion metal $\mathrm{UTe}_{2}$, Nature (London) 579, 523 (2020).

[44] I. Titvinidze, A. Schwabe, and M. Potthoff, Ferromagnetism of magnetic impurities coupled indirectly via conduction electrons: Insights from various theoretical approaches, Phys. Rev. B 90, 045112 (2014).

[45] A. Schwabe, I. Titvinidze, and M. Potthoff, Inverse indirect magnetic exchange, Phys. Rev. B 88, 121107 (2013).

[46] N. C. Costa, M. V. Araújo, J. P. Lima, T. Paiva, R. R. dos Santos, and R. T. Scalettar, Compressible ferrimagnetism in the depleted periodiC anderson model, Phys. Rev. B 97, 085123 (2018).

[47] A. Ramires and J. L. Lado, Impurity-induced triple point fermions in twisted bilayer graphene, Phys. Rev. B 99, 245118 (2019).

[48] A. Lopez-Bezanilla and J. L. Lado, Defect-induced magnetism and Yu-Shiba-Rusinov states in twisted bilayer graphene, Phys. Rev. Materials 3, 084003 (2019).
[49] M. A. Ruderman and C. Kittel, Indirect exchange coupling of nuclear magnetic moments by conduction electrons, Phys. Rev. 96, 99 (1954)

[50] K. Yosida, Magnetic properties of Cu-Mn alloys, Phys. Rev. 106, 893 (1957).

[51] T. Kasuya, A theory of metallic ferro- and antiferromagnetism on Zener's model, Prog. Theor. Phys. 16, 45 (1956).

[52] B. Sutherland, Localization of electronic wave functions due to local topology, Phys. Rev. B 34, 5208 (1986).

[53] P. W. Brouwer, E. Racine, A. Furusaki, Y. Hatsugai, Y. Morita, and C. Mudry, Zero modes in the random hopping model, Phys. Rev. B 66, 014204 (2002).

[54] A. Mielke, Ferromagnetism in the Hubbard model on line graphs and further considerations, J. Phys. A: Math. Gen. 24, 3311 (1991).

[55] E. H. Lieb, Two Theorems on the Hubbard Model, Phys. Rev. Lett. 62, 1201 (1989).

[56] P. Fazekas and E. Müller-Hartmann, Magnetic and nonmagnetic ground states of the Kondo lattice, Z. Phys. B 85, 285 (1991).

[57] S. Doniach, The Kondo lattice and weak antiferromagnetism, Physica B+C 91, 231 (1977).

[58] D. G. Mazzone, N. Gauthier, D. T. Maimone, R. Yadav, M Bartkowiak, J. L. Gavilano, S. Raymond, V. Pomjakushin, N. Casati, Z. Revay, G. Lapertot, R. Sibille, and M. Kenzelmann, Evolution of Magnetic Order from the Localized to the Itinerant Limit, Phys. Rev. Lett. 123, 097201 (2019).

[59] S. Jang, R. Kealhofer, C. John, S. Doyle, J.-S. Hong, J. H. Shim, Q. Si, O. Erten, J. D. Denlinger, and J. G. Analytis, Direct visualization of coexisting channels of interaction in $\mathrm{CeSb}$, Sci. Adv. 5, eaat7158 (2019).

[60] Y. Liu, G.-M. Zhang, and L. Yu, Weak ferromagnetism with the Kondo screening effect in the Kondo lattice systems, Phys. Rev. B 87, 134409 (2013).

[61] R. Peters and N. Kawakami, Competition of striped magnetic order and partial Kondo screened state in the Kondo lattice model, Phys. Rev. B 96, 115158 (2017).

[62] M. W. Aulbach, F. F. Assaad, and M. Potthoff, Dynamical mean-field study of partial Kondo screening in the periodic anderson model on the triangular lattice, Phys. Rev. B 92, 235131 (2015).

[63] S. Capponi and F. F. Assaad, Spin and charge dynamics of the ferromagnetic and antiferromagnetic two-dimensional half-filled Kondo lattice model, Phys. Rev. B 63, 155114 (2001).

[64] H. Watanabe and M. Ogata, Fermi-Surface Reconstruction Without Breakdown of Kondo Screening at the Quantum Critical Point, Phys. Rev. Lett. 99, 136401 (2007).

[65] L. Isaev and I. Vekhter, Heavy Antiferromagnetic Phases in Kondo Lattices, Phys. Rev. Lett. 110, 026403 (2013).

[66] R. Peters, N. Kawakami, and T. Pruschke, Spin-Selective Kondo Insulator: Cooperation of Ferromagnetism and the Kondo Effect, Phys. Rev. Lett. 108, 086402 (2012).

[67] A. Georges, G. Kotliar, W. Krauth, and M. J. Rozenberg, Dynamical mean-field theory of strongly correlated fermion systems and the limit of infinite dimensions, Rev. Mod. Phys. 68, 13 (1996).

[68] F. F. Assaad and T. C. Lang, Diagrammatic determinantal quantum monte carlo methods: Projective schemes and 
applications to the Hubbard-Holstein model, Phys. Rev. B 76, 035116 (2007).

[69] E. Gull, Continuous-time quantum Monte Carlo algorithms for fermions, Ph.D. thesis, ETH Zruich (2008).

[70] T. I. Vanhala and P. Törmä, Dynamical mean-field theory study of stripe order and $d$-wave superconductivity in the twodimensional hubbard model, Phys. Rev. B 97, 075112 (2018).

[71] A. I. Lichtenstein and M. I. Katsnelson, Antiferromagnetism and d-wave superconductivity in cuprates: A cluster dynamical mean-field theory, Phys. Rev. B 62, R9283 (2000).

[72] M. Capone and G. Kotliar, Competition between $d$-wave superconductivity and antiferromagnetism in the two-dimensional Hubbard model, Phys. Rev. B 74, 054513 (2006).

[73] S. Burdin and P. Fulde, Random Kondo alloys investigated with the coherent potential approximation, Phys. Rev. B 76, 104425 (2007).

[74] J. E. Hirsch, Two-dimensional Hubbard model: Numerical simulation study, Phys. Rev. B 31, 4403 (1985).

[75] K. Noda, A. Koga, N. Kawakami, and T. Pruschke, Ferromagnetism of cold fermions loaded into a decorated square lattice, Phys. Rev. A 80, 063622 (2009).
[76] K. Noda, K. Inaba, and M. Yamashita, Magnetism in the three-dimensional layered lieb lattice: Enhanced transition temperature via flat-band and Van Hove singularities, Phys. Rev. A 91, 063610 (2015).

[77] N. D. Mermin and H. Wagner, Absence of Ferromagnetism or Antiferromagnetism in One- or Two-Dimensional Isotropic Heisenberg Models, Phys. Rev. Lett. 17, 1133 (1966).

[78] P. C. Hohenberg, Existence of long-range order in one and two dimensions, Phys. Rev. 158, 383 (1967).

[79] L. Fratino, P. Sémon, M. Charlebois, G. Sordi, and A.M. S. Tremblay, Signatures of the Mott transition in the antiferromagnetic state of the two-dimensional hubbard model, Phys. Rev. B 95, 235109 (2017).

[80] S. R. White, Density Matrix Formulation for Quantum Renormalization Groups, Phys. Rev. Lett. 69, 2863 (1992).

[81] M. Fishman, S. R. White, and E. M. Stoudenmire, The ITensor Software Library for Tensor Network Calculations, arXiv:2007.14822 [cs.MS].

[82] ITensor Library http://itensor.org.

[83] DMRGpy Library https://github.com/joselado/dmrgpy. 